\title{
Structure of the profilin-poly-L-proline complex involved in morphogenesis and cytoskeletal regulation
}

\author{
Nicole M. Mahoney, Paul A. Janmey and Steven C. Almo \\ Nature Struct. Biol. 4, 953-960 (1997)
}

Due to a printer's error, the bottom line ('Birch pollen') of the sequence alignment in Fig. 3 was lost and the bottom edge of the image in Fig. 6 was cropped. The versions below are how the figures should have appeared.

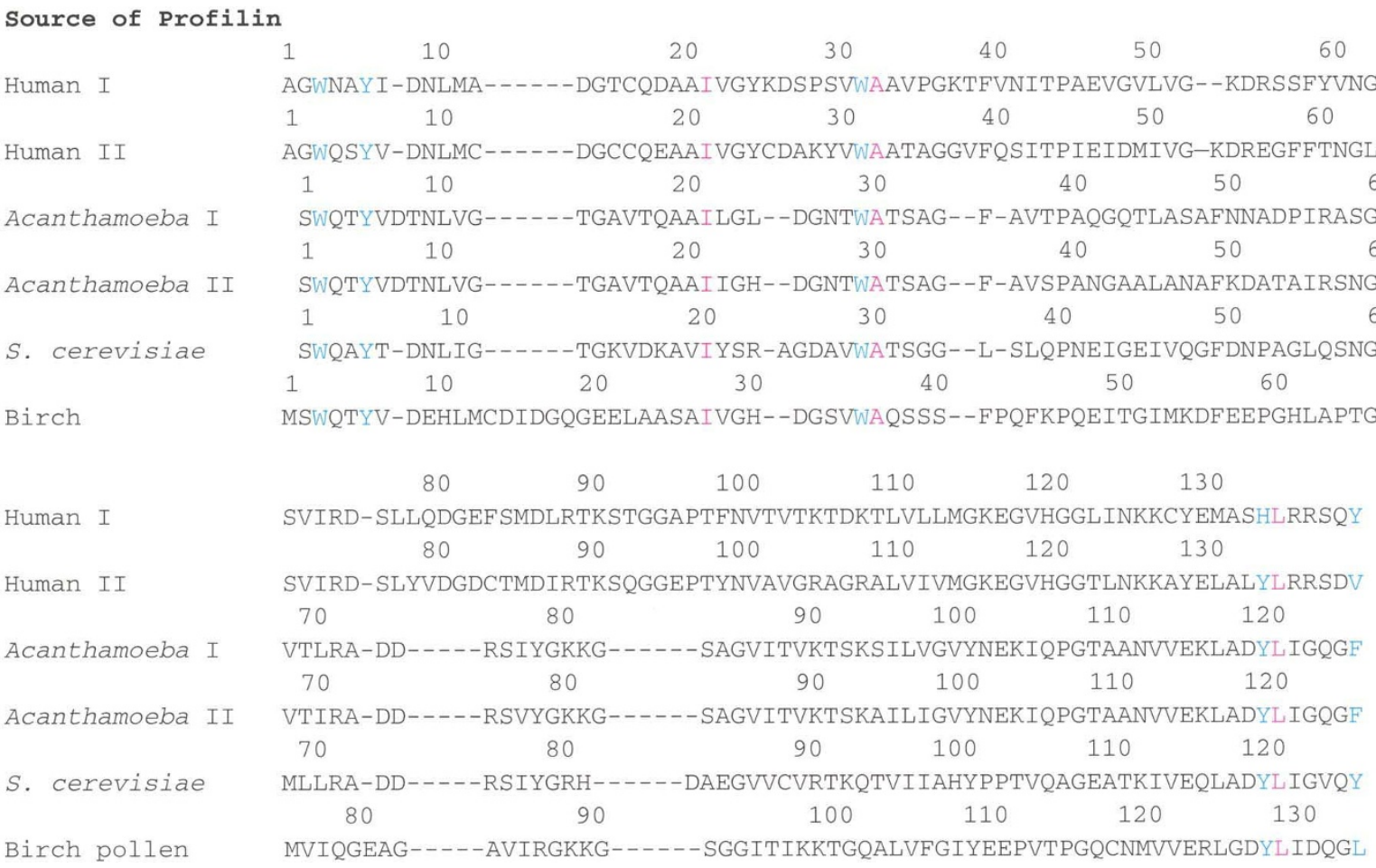

Fig. 3 Structure based sequence alignment of human profilin I (that is, human platelet profilin), human profilin II, Acanthamoeba profilin I, Acanthamoeba profilin I, Saccharomyces cerevisiae profilin, and birch pollen profilin, adapted in part from ref. 53 . Amino acids occupying equivalent positions were aligned after the superposition of the refined models. Blue residues correspond to amino acids directly involved in poly-L-proline binding, while red residues are involved in packing and the proper positioning of the actual contact residues.

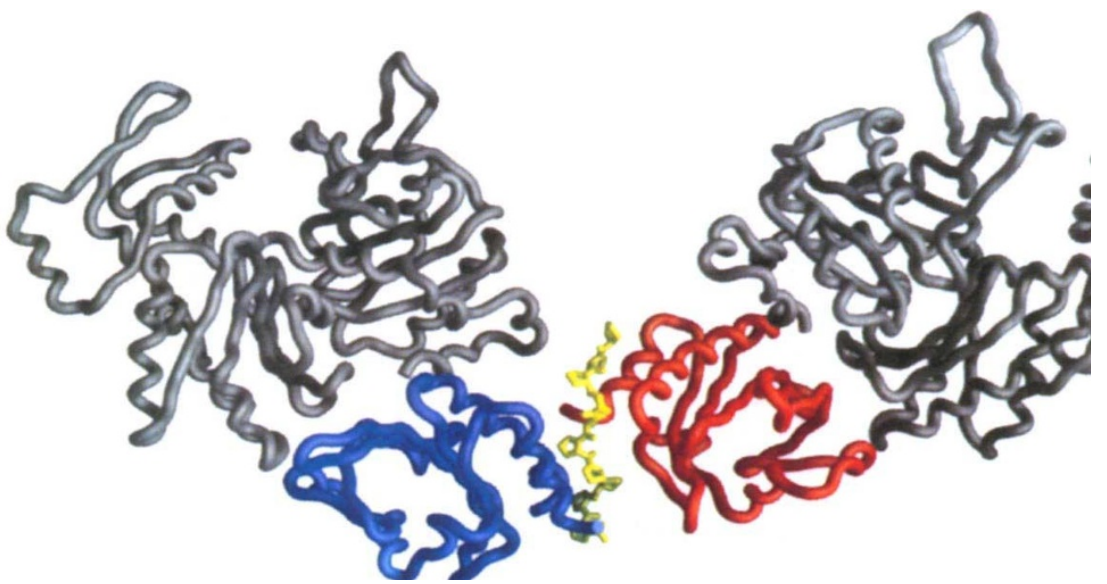

Fig. 6 Model of the profilin-actin-peptide complex showing that the poly-L-proline and actin binding sites are independent and non-interacting (profilin in red and blue, actin in gray, peptide in yellow). This is consistent with the simultaneous binding of actin and proline-rich peptides by profilin, which is required for site specific F-actin assembly. This model was generated by superimposing the bovine profilin actin complex ${ }^{26}$ on the HPP-L-Pro ${ }_{10}$ coordinates based on $C \alpha$ atoms of the profilin molecules. This figure was generated using GRASP51. 